\title{
Long Decays of Excitonic Photoluminescence from CdTe/ZnTe Individual Quantum Dots
}

\author{
B. Piechal, J. Suffczyński, A. Trajnerowicz, M. Goryca, \\ P. Kossacki, A. Golnik and J.A. GaJ
}

Institute of Experimental Physics, Warsaw University

Hoża 69, 00-681 Warsaw, Poland

\begin{abstract}
We present time-resolved micro-photoluminescence study of self-assembled $\mathrm{CdTe} / \mathrm{ZnTe}$ quantum dots. In addition to the fast radiative decay of the exciton, we observe long components, being of the order of a few nanoseconds. We observe that long decays are present for neutral exciton lines as well as on the biexciton ones. The temperature dependent measurements reveal the non-monotonic behaviour. We find also that the long decays are not mono-exponential. We conclude that the dark exciton states cannot be treated as the only source of long decays in the luminescence. We propose other possible explanation, which is the slowing of luminescence by carrier trapping in high energy states in quantum dots and barriers.
\end{abstract}

PACS numbers: 78.47.+p, 78.67.Hc, 78.55.Et

\section{Introduction}

Semiconductor quantum dots (QDs) have recently become the subject of intense investigation as a possible source of the triggered entangled photons. The efforts were originally focused on the classical InAs/GaAs QD system [1, 2]. Subsequently, II-VI systems have been receiving more attention because of their more robust excitons, observable at higher temperatures. Within this area the observation of bunching and antibunching effects under continuous wave excitation was reported for $\mathrm{CdTe} / \mathrm{ZnTe} \mathrm{QDs}$ [3]. The same paper reports the dynamics measurements that revealed biexponential decay of the luminescence of quantum dots. The short component of the decay represents the radiative lifetime of the bright exciton state, being of the order of 200 ps. The long one, being of the order of a few nanoseconds was interpreted as due to the dark exciton state. In such case the long decay would derive from the thermally activated spin flip of the carrier in 
the dark exciton state. However, further investigations were suggested to confirm the interpretation.

In order to verify the above hypothesis the time-resolved micro-photoluminescence was studied as a function of sample temperature. Our measurements were performed on the samples, one of them being the same sample, as in Ref. [3], but the excitation conditions and temperatures were different.

\section{Experiment}

Two samples were investigated. The first one contained CdTe QDs embedded in $\mathrm{Zn}(\mathrm{Mg}) \mathrm{Te}$ barriers. It was the sample studied in [3] and described in detail in [4]. It will be called "\#1" The second one (let us call it "\#2") contained the CdTe self-assembled QDs in ZnTe barriers. This sample was grown by MBE technique. On the (001) oriented GaAs substrate a $4 \mu \mathrm{m}$ CdTe buffer has been deposited and followed by $1 \mu \mathrm{m}$ ZnTe barrier. The QDs consisted of 2 monolayers of CdTe and were capped by $0.5 \mu \mathrm{m}$ of ZnTe. The spectral range of the QD-emission of the sample \#1 is between 550 and $660 \mathrm{~nm}(1.87-2.25 \mathrm{eV})$, while that of the sample \#2 is between 530 and $560 \mathrm{~nm}(2.20-2.35 \mathrm{eV})$.

Each sample was mounted directly on a mirror microscope objective [5] and placed inside a pumped helium cryostat. The temperature was changed between 5 and $50 \mathrm{~K}$. The luminescence was excited by 85 ps laser pulses (wavelength $405 \mathrm{~nm}$ ). These were generated by a semiconductor laser diode operating at a repetition rate of $20 \mathrm{MHz}$. The same microscope objective was used to focus laser light on the sample as well as to collect the luminescence, which was then dispersed by a monochromator with an 1800 grooves/mm grating. The emission spectra were measured with a CCD camera.

The dynamics of the luminescence was detected with a micro-channel plate (MCP) photomultiplier, or alternatively a standard photomultiplier (PMT). The signal from each was collected by a Pico-Quant Time Harp 200 fast photon counting card mounted in the PC computer. The temporal resolution of the detectors is $55 \mathrm{ps}$ in case of MCP photomultiplier and $850 \mathrm{ps}$ in case of PMT. Therefore MCP was used whenever the emission was in its spectral range. That was the case of the lines from long-wave tail of the emission spectra of the sample \#2 and short-wave side of the spectra of sample \#1. The luminescence lines from long-wave tail of the spectra of the sample \#1 were detected using PMT.

\section{Spectra and line identification}

Figure 1a shows emission spectra of a single QD measured on the sample \#2. The three lines shown in the figure are assigned to the same quantum dot with the following arguments.

First, all the three lines are the luminescence coming from the same point on the sample, within our spatial resolution. This means that as we were moving 

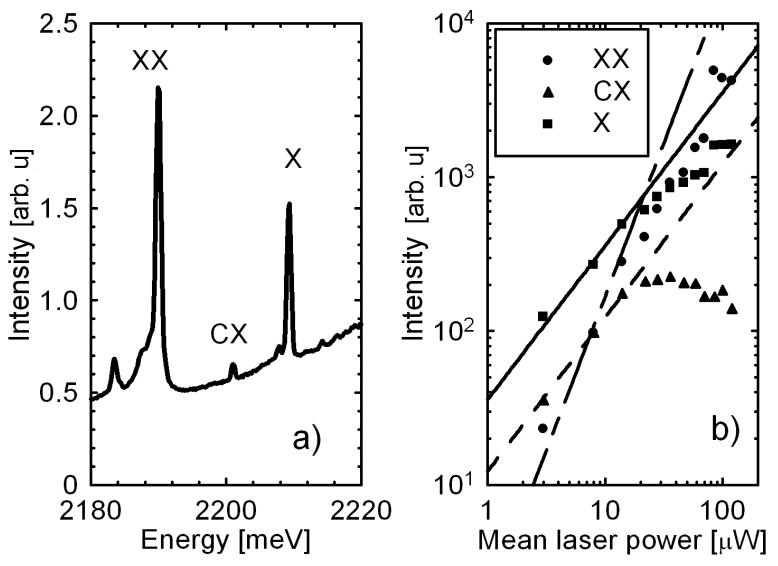

Fig. 1. Spectrum of single quantum dot (a) and intensity of the lines versus excitation power (b).

the laser spot over the surface of the sample, the lines appeared and disappeared synchronically.

Secondly, all the triples of the lines found this way had a similar structure two strong lines separated by about $20 \mathrm{meV}$ from each other and the third weak one between them.

Finally, the dependence of the line intensities on excitation power reveals the quadratic behaviour of the low energy line (at $2190 \mathrm{meV}$ in Fig. 1a, $X X$ ) and linear behaviour of the two other ones $(X$ and $C X)$. It is shown in Fig. 1b. The quadratic dependence on the excitation power of the $X X$ line allows us to identify it as biexciton line [6]. Similar triples have been identified in the same sample by the same arguments, completed by in-plane optical anisotropy and Zeeman effect measurements [7].

On the sample \#1 we were able to identify only single lines from each QD, attributed to neutral exciton $(X)$ recombination.

\section{Results and discussion}

In Ref. [3] the time-resolved photoluminescence study of CdTe/ZnTe QDs revealed two components in decay curves of luminescence. The long one, being of the order of a few nanoseconds, was interpreted tentatively as due to the presence of dark exciton state.

Figure 2a shows examples of the time-resolved luminescence of the $X$ line at various temperatures. Figure $2 \mathrm{~b}$ shows the long component decay time plotted as a function of temperature. The presented measurements were performed on the sample studied in [3] (i.e. sample \#1 - open symbols in Fig. 2b), as well as on the sample \#2 (closed symbols). We observe the presence of the long decays on both samples. Our measurements reveal in some cases a non-monotonic temperature 

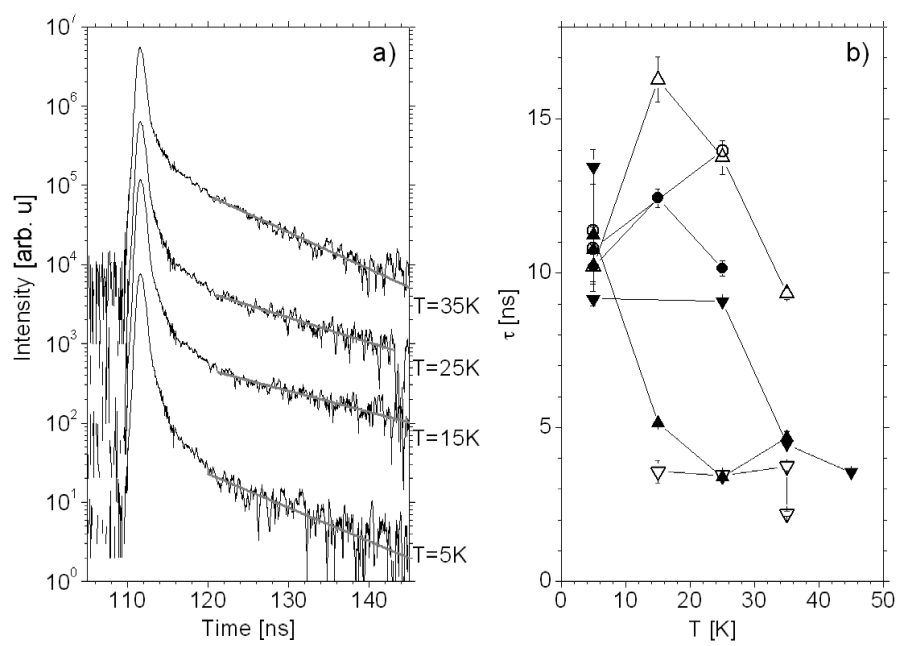

Fig. 2. Time-resolved photoluminescence at various temperatures (a) and temperature dependence of long decay time (b). Open symbols are for the data obtained on the sample \#1, closed symbols - on the sample \#2.

dependence of the long decay times. At temperatures of over $25 \mathrm{~K}$ the long decay time decreases with temperature. However, at temperature of $5 \mathrm{~K}$ the decay time is often shorter than at temperature of $15 \mathrm{~K}$. This is not expected within the hypothesis of the dark exciton origin of the long decays, where the decay time should exhibit activation behaviour.

Another observation contradictory to the hypothesis of dark exciton origin of the long decays in luminescence is the presence of these decays not only for the neutral exciton line, but also for the biexciton one: the dark exciton population can hardly feed the biexciton luminescence. It is clearly seen in Fig. 3.

The last point we would like to discuss is the actual shape of a luminescence decay. Trials to fit a double exponent function to the experimental decay data fail showing that the light emission decay is not actually biexponential (Fig. 4 dashed line).

All the observations reported above suggest that the dark exciton state should not be considered as the only source of the long decays of luminescence. Another mechanism might be related to slow relaxation from high-energy states in other quantum dots and barriers. This mechanism, contrary to the dark exciton one, should result in long decay components of emission not only for the neutral exciton state, but also for the biexciton one. Also, the long decay in this case instead of being exponential, should rather have the following form $[8,9]$ :

$$
I(t)=A \exp \left(\left(\frac{t-t_{0}}{\tau^{\prime}}\right)^{\delta}\right) .
$$

The solid line in Fig. 4 presents the fit of the function being the sum of fast 


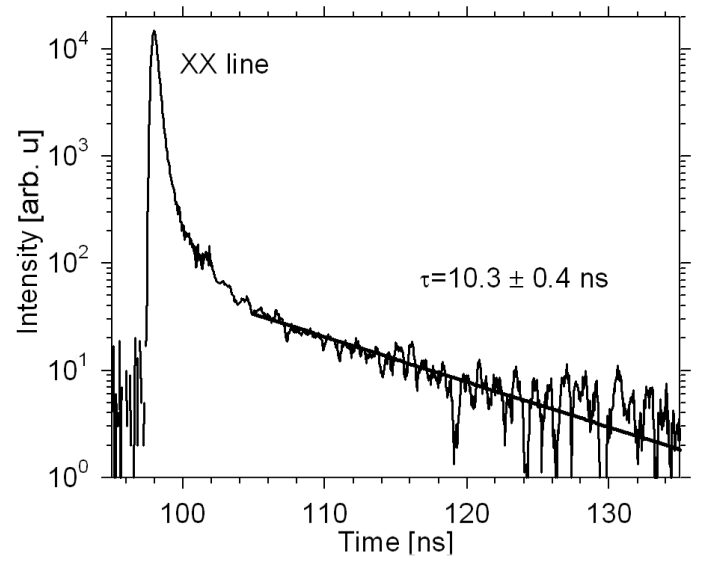

Fig. 3. Time-resolved photoluminescence measured at the biexciton line. The long decays are observed not only for excitonic lines, but also for biexcitonic ones.

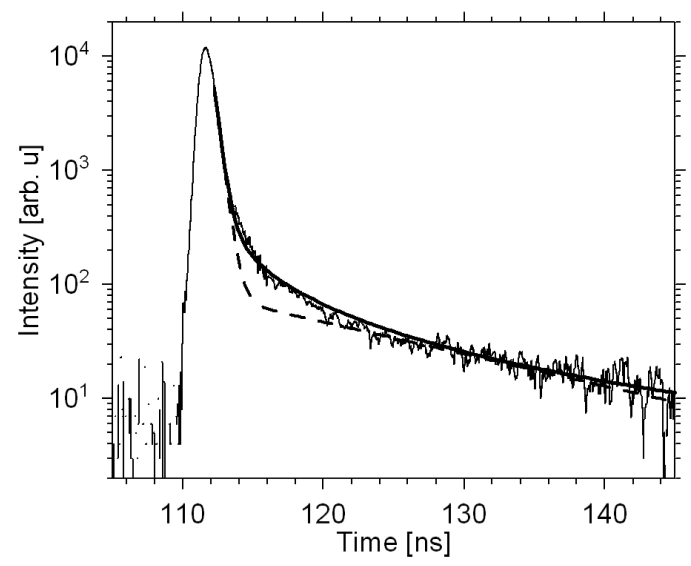

Fig. 4. Time-resolved luminescence of excitonic line. Fitted function represents various mechanisms of the long decays. Dashed line is the double exponential decay. Solid line represents the function from Eq. (1) (fit obtained with $\delta=0.2$ ).

exponential decay and slower decay described by Eq. (1). Such model represents the luminescence shape much better than the biexponential function.

\section{Conclusions}

The temperature dependence of the long decay rates of the exciton lines, as well as the observation of the long component in biexcitonic decays show that the dark exciton state cannot be treated as the only source of the nanosecond-long decays of the excitonic photoluminescence from quantum dots. Another possible origin is a slow relaxation of photoexcited carriers from high energy states in other dots and barriers. 


\section{Acknowledgments}

We express our gratitude to Henri Mariette and Frank Tinjod from CEA Grenoble and Grzegorz Karczewski and Jacek Kossut from IFPAN Warsaw for samples and to Jean-Philippe Poizat for lending us a micro-channel plate photomultiplier.

This work was supported by the State Committee for Scientific Research grants PBZ-KBN-044/P03/2001 and 2 P03B 00225) (Poland).

\section{References}

[1] C. Santori, M. Pelton, G. Solomon, Y. Dale, Y. Yamamoto, Phys. Rev. Lett. 86, 1502 (2001).

[2] E. Moreau, I. Robert, L. Manin, V. Thierry-Mieg, J.M. Gérard, I. Abram, Phys. Rev. Lett. 87, 183601 (2001).

[3] C. Couteau, S. Moehl, F. Tinjod, J.M. Gérard, K. Kheng, H. Mariette, J.A. Gaj, R. Romestain, J.P. Poizat, Appl. Phys. Lett. 85, 6251 (2004).

[4] F. Tinjod, B. Gilles, S. Moehl, K. Kheng, H. Mariette, Appl. Phys. Lett. 82, 4340 (2003).

[5] J. Jasny, J. Sepioł, Chem. Phys. Lett. 273, 439 (1997).

[6] L. Besombes, K. Kheng, L. Marsal, H. Mariette, Phys. Rev. B 65, 121314 (2002).

[7] A. Kudelski, K. Kowalik, A. Golnik, G. Karczewski, J. Kossut, J.A. Gaj, J. Lumin. 112, 127 (2005).

[8] R.G. Palmer, D.L. Stein, E. Abrahams, P.W. Anderson, Phys. Rev. Lett. 53, 958 (1984).

[9] R. Kohlrausch, Ann. Phys. (Leipzig) 12, 393 (1947). 